\title{
Parton distribution functions (PDFs) from dipole models and HERA data - predictions for the LHC
}

\author{
Agnieszka Luszczak ${ }^{1, a}$ \\ ${ }^{1}$ T.Kosciuszko Cracow University of Technology
}

\begin{abstract}
We analyse, within a dipole model, the final, inclusive HERA DIS cross section data in the low $x$ region, using fully correlated errors. We show, that these highest precision data are very well described within the dipole model framework starting from $Q^{2}$ values of $3.5 \mathrm{GeV}^{2}$ to the highest values of $Q^{2}=250 \mathrm{GeV}^{2}$. We made predictions for the LHC data using parton distributions functions (PDFs) in particular - gluon distributions from BGK dipole model fits to HERA data
\end{abstract}

\section{Introduction}

Many investigations have shown that HERA inclusive and diffractive DIS cross sections are very well described by the dipole models [1-3], which provide a natural description of QCD reaction in the low$x$ and low $Q^{2}$ region. They allow a simultaneous description of many different physics reactions, like inclusive DIS processes, inclusive diffractive processes, exclusive $J / \psi, \rho, \phi$ production, diffractive jet production, or diffractive and non-diffractive charm production. Due to the optical theorem, all these processes are determined by the same, universal, gluon density [4-6]. The understanding of the properties of the gluon density and its precise knowledge is very important because the QCD-evolved gluon density determines the cross sections of most relevant physics processes, e.g. Higgs production at LHC. Any significant deviation of the predicted cross section from their Standard Model value could be a sign of new physics.

The precise determination of the gluon density relies on the analysis of high quality, inclusive, DIS data taken over the full, experimentally accessible $x$ and $Q^{2}$ region. Therefore, the H1 and ZEUS experiments have combined their inclusive DIS cross sections which, due to a substantial reduction of systematic measurements errors, led to an increase of precision by about a factor two [7]. More recently, also the final set of HERA data was released which provides a full set of correlated errors. These errors contain the most complete information about the data and lead to very restrictive fits [8]. The full information about the data is made now accessible within the xFitter facility [9].

\section{Dipole models}

The dipole picture was first derived, in the low $x$ limit of QCD, by Nikolaev and Zaharov [10]. They have shown that the deep inelastic scattering can be viewed as a two stage process; first the

\footnotetext{
ae-mail: agnieszka.luszczak@ifj.edu.pl
} 
virtual photon fluctuates into a dipole, which consists of a quark-antiquark pair (or a $q \bar{q} g$ or $q \bar{q} g g$... system) and in the second stage the dipole interacts with the proton. Dipole denotes a quasi-stable quantum mechanical state, which has a very long life time $\left(\approx 1 / m_{p} x\right)$ and a size $r$, which remains unchanged during scattering. The wave function $\Psi$ determines the probability to find a dipole of size $r$ within a photon. This probability depends on the value of external $Q^{2}$ and the fraction of the photon momentum carried by the quarks forming the dipole, $z$. Neglecting the $z$ dependence, in a very rough approximathion, $Q^{2} \sim 1 / r^{2}$.

The scattering amplitude is a product of the virtual photon wave function, $\Psi$, with the dipole cross section, $\sigma_{\text {dip }}$, which determines a probability of the dipole-proton scattering. Thus, within the dipole formulation of the $\gamma^{*} p$ scattering

$$
\sigma_{T, L}^{\gamma^{*} p}\left(x, Q^{2}\right)=\int d r^{2} \int d z \Psi_{T, L}^{*}(Q, r, z) \sigma_{\mathrm{dip}}(x, r) \Psi_{T, L}(Q, r, z),
$$

where $T, L$ denotes the virtual photon polarization and $\sigma_{T, L}^{\gamma^{*} p}$ the total inclusive DIS cross section.

This simple and intuitive approach became then a basis of many dipole many models, [11-17]. which have been developed to test various aspects of data. They vary due to different assumption made about the physical behavior of dipole cross sections. In the following we will shortly review some them to motivate the choice of the model used for present investigation.

\subsection{GBW model}

The dipole model became an important tool in investigations of deep-inelastic scattering due to the initial observation of Golec-Biernat and Wüesthoff (GBW) [1], that a simple ansatz for the dipole cross section was able to describe simultaneously the total inclusive and diffractive cross sections.

In the GBW model the dipole-proton cross section $\sigma_{\text {dip }}$ is given by

$$
\sigma_{\text {dip }}\left(x, r^{2}\right)=\sigma_{0}\left(1-\exp \left[-\frac{r^{2}}{4 R_{0}^{2}(x)}\right]\right),
$$

where $r$ corresponds to the transverse separation between the quark and the antiquark, and $R_{0}^{2}$ is an $x$ dependent scale parameter which has a meaning of saturation radius, $R_{0}^{2}(x)=\left(x / x_{0}\right)^{\lambda_{G B W}} / G e V^{-2}$. The free fitted parameters are: the cross-section normalisation, $\sigma_{0}$, as well as $x_{0}$ and $\lambda_{G B W}$. In this model saturation is taken into account in the eikonal approximation and the saturation radius is intimately related to the gluon density, see below. The exponent $\lambda_{G B W}$ determines the growth of the total and diffractive cross section with decreasing $x$. For dipole sizes which are large in comparison to the saturation radius, $R_{0}$, the dipole cross section saturates by approaching a constant value $\sigma_{0}$, i.e. saturation damps the growth of the gluon density at low $x$.

The GBW model provided a good description of data from medium $Q^{2}$ values $\left(\approx 30 \mathrm{GeV}^{2}\right)$ down to low $\left.Q^{2}(\approx 0.1) \mathrm{GeV}^{2}\right)$. Despite its success and its appealing simplicity the model has some shortcomings; in particular it describes the QCD evolution by a simple $x$ dependence, $\sim(1 / x)_{B G W}^{\lambda}$, i.e the $Q^{2}$ dependence of the cross section evolution is solely induced by the saturation effects. Therefore, it does not match with DGLAP QCD evolution, which is known to describe data very well from $Q^{2} \approx 4 \mathrm{GeV}^{2}$ to very large $Q^{2} \approx 10000 \mathrm{GeV}^{2}$.

\subsection{BGK model}

The evolution ansatz of the GBW model was improved in the model proposed by Bartels, GolecBiernat and Kowalski, (BGK) [2], by taking into account the DGLAP evolution of the gluon density 
in an explicit way. The model preserves the GBW eikonal approximation to saturation and thus the dipole cross section is given by

$$
\sigma_{\text {dip }}\left(x, r^{2}\right)=\sigma_{0}\left(1-\exp \left[-\frac{\pi^{2} r^{2} \alpha_{s}\left(\mu^{2}\right) x g\left(x, \mu^{2}\right)}{3 \sigma_{0}}\right]\right) .
$$

The evolution scale $\mu^{2}$ is connected to the size of the dipole by $\mu^{2}=C / r^{2}+\mu_{0}^{2}$. This assumption allows to treat consistently the contributions of large dipoles without making the strong coupling constant, $\alpha_{s}\left(\mu^{2}\right)$, un-physically large. This means also that we can extend the model, keeping its perturbative character, to the data at low $Q^{2}$, because the external $Q^{2}$ and the internal $\mu^{2}$ scales are connected only by the wave function.

The gluon density, which is parametrized at the starting scale $\mu_{0}^{2}$, is evolved to larger scales, $\mu^{2}$, using LO or NLO DGLAP evolution. We consider here two forms of the gluon density:

- the soft ansatz, as used in the original BGK model

$$
x g\left(x, \mu_{0}^{2}\right)=A_{g} x^{-\lambda_{g}}(1-x)^{C_{g}},
$$

- the soft + hard ansatz

$$
x g\left(x, \mu_{0}^{2}\right)=A_{g} x^{-\lambda_{g}}(1-x)^{C_{g}}\left(1+D_{g} x+E_{g} x^{2}\right),
$$

The free parameters for this model are $\sigma_{0}$ and the parameters for gluon $A_{g}, \lambda_{g}, C_{g}$ or additionally $D_{g}, E_{g}$, Their values are obtained by a fit to the data. The fit results were found to be independent on the parameter $C$, which was therefore fixed as $C=4 \mathrm{GeV}^{2}$, in agreement with the original BGK fits. It is also possible to vary the parameter $\mu_{0}^{2}$. However, to assure that the evolution is performed in the perturbative region and to be compatible with the standard pdf fits we took as a starting scale $\mu_{0}^{2}=1.9$ or $1.1 \mathrm{GeV}^{2}$. In the BGK model, the $\mu_{0}^{2}$ scale is the same as the $Q_{0}^{2}$ scale of the standard QCD pdf fits.

\section{Fits to the dipole model}

The results of the BGK fit with valence quarks and the soft gluon density, are shown in Table 1, 2 and 3 . The best fit is obtained using valence quarks and gluon density of the soft + hard type, see Table 4 and 6. In Table 1, 2 and 4 the starting QCD scale is $Q_{0}^{2}=1.9 \mathrm{GeV}^{2}$, in Table 6 it is $Q_{0}^{2}=1.1 \mathrm{GeV}^{2}$,

In Table 1, 4 and 6 the valence quark contribution was taken from the standard pdf fit, shown below, in Table 2 the valence quarks were fitted. In these tables, $N_{d f}$ denotes the number of degrees of freedom, which is equal to the number of measured data points minus the number of free parameters used in the fit. The parameters $\sigma_{0}$ of the dipole model and the starting parameters for gluon $A_{g}, \lambda_{g}$, $C_{g}$ are obtained from the fit. The value of the parameter $C$ was fixed, as explained above. To limit the fit to the perturbative region only we took $Q^{2} \geq Q_{\min }^{2}$ with $Q_{\min }^{2}=3.5$ (or 8.5) $\mathrm{GeV}^{2}$. For the $x$ region, we took $x \leq 0.01$. There are 538 (or 452) measured points in this region. 


\begin{tabular}{|c|c|c|c|c|c|c|c|}
\hline$Q_{\min }^{2}\left[\mathrm{GeV}^{2}\right]$ & $\sigma_{0}[\mathrm{mb}]$ & $A_{g}$ & $\lambda_{g}$ & $C_{g}$ & $N_{d f}$ & $\chi^{2}$ & $\chi^{2} / N_{d f}$ \\
\hline 3.5 & $87.0 \pm$ & $2.32 \pm$ & $-0.056 \pm$ & $8.21 \pm$ & 534 & 551.1 & 1.03 \\
& 8.9 & 0.009 & 0.11 & 0.80 & & & \\
8.5 & $72.4 \pm$ & $2.77 \pm$ & $-0.042 \pm$ & $6.54 \pm$ & 448 & 452.5 & 1.01 \\
& 7.4 & 0.009 & 0.123 & 0.632 & & & \\
\hline
\end{tabular}

Table 1. BGK fit with fixed valence quarks for $\sigma_{r}$ for H1ZEUS-NC data in the range $Q^{2} \geq 3.5$ or $8.5 \mathrm{GeV}^{2}$ and $x \leq 0.01$. NLO fit. Soft gluon. $m_{u d s}=0.14, m_{c}=1.3 \mathrm{GeV} . Q_{0}^{2}=1.9 \mathrm{GeV}^{2}$.

\begin{tabular}{|c|c|c|c|c|c|c|c|}
\hline$Q_{\min }^{2}\left[\mathrm{GeV}^{2}\right]$ & $\sigma_{0}[\mathrm{mb}]$ & $A_{g}$ & $\lambda_{g}$ & $C_{g}$ & $N_{d f}$ & $\chi^{2}$ & $\chi^{2} / N_{d f}$ \\
\hline 3.5 & $89.99 \pm$ & $2.44 \pm$ & $-0.079 \pm$ & $7.24 \pm$ & 530 & 540.35 & 1.02 \\
& 9.2 & 0.145 & 0.099 & 0.61 & & & \\
\hline
\end{tabular}

Table 2. BGK fit with fitted valence quarks for $\sigma_{r}$ for H1ZEUS-NC data in the range $Q^{2} \geq 3.5 \mathrm{GeV}^{2}$ and $x \leq 0.01$. NLO fit. Soft gluon. $m_{u d s}=0.14, m_{c}=1.3 \mathrm{GeV} . Q_{0}^{2}=1.9 \mathrm{GeV}^{2}$.

\begin{tabular}{|c||c||c||c|c||c|c|c||}
\hline No & $A u v$ (fix) & $B u v$ & $C u v$ & $E u v$ & $A d v(f i x)$ & $B d v$ & $C d v$ \\
\hline 1 & 4.073 (sum rule) & $0.892 \pm$ & $5.832 \pm$ & $17.997 \pm$ & 3.151 (sum rule) & $0.840 \pm$ & $3.480 \pm$ \\
& & 0.019 & 0.341 & 0.876 & & 0.012 & 0.056 \\
\hline
\end{tabular}

Table 3. Parameters of the valence quark contribution fitted in the BGK fit of Table 2.

\begin{tabular}{|c|c|c|c|c|c|c|c|c|c|}
\hline$Q_{\min }^{2}\left[\mathrm{GeV}^{2}\right]$ & $\sigma_{0}[\mathrm{mb}]$ & $A_{g}$ & $\lambda_{g}$ & $C_{g}$ & $D_{g}$ & $E_{g}$ & $N_{d f}$ & $\chi^{2}$ & $\chi^{2} / N_{d f}$ \\
\hline 3.5 & $77,6 \pm$ & $2.62 \pm$ & $-0.064 \pm$ & $37.1 \pm$ & $3.06 \pm$ & $1406.4 \pm$ & 532 & 534.2 & 1.00 \\
& 18,6 & 0.16 & 0.0087 & 5.06 & 6.51 & 552.7 & & & \\
\hline 8.5 & $63.5 \pm$ & $2.11 \pm$ & $-0.054 \pm$ & $21.3 \pm$ & $1.10 \pm$ & $867.2 \pm$ & 448 & 439.0 & 0.98 \\
& 18.5 & 0.10 & 0.0065 & 4.062 & 5.76 & 423.7 & & & \\
\hline
\end{tabular}

Table 4. BGK fit with valence quarks for $\sigma_{r}$ for H1ZEUS-NC data in the range $Q^{2} \geq 3.5$ or $8.5 \mathrm{GeV}^{2}$ and $x \leq 0.01$. NLO fit. Soft + hard gluon. $m_{u d s}=0.14, m_{c}=1.3 \mathrm{GeV} . Q_{0}^{2}=1.9 \mathrm{GeV}^{2}$.

\section{Gluon density from BGK dipole model}

In Figure 1 we compare the gluon densities obtained from fits with and without saturation ansatz of Tables 4 and 5. Note that for $x<0.001$, the gluon densities obtained in the dipole approach are higher than that of the standard pdf fit, and that of the non-saturated one, see Fig 1. This is expected and is due to damping of gluon density by saturation effects. 


\begin{tabular}{|c|c|c|c|c|c|c|c|c|c|}
\hline$Q_{\min }^{2}\left[\mathrm{GeV}^{2}\right]$ & $Q_{0}^{2}\left[\mathrm{GeV}^{2}\right]$ & $A_{g}$ & $\lambda_{g}$ & $C_{g}$ & $D_{g}$ & $E_{g}$ & $N d f$ & $\chi^{2}$ & $\chi^{2} / N p$ \\
\hline 3.5 & 1.9 & $2.33 \pm$ & $-0.094 \pm$ & $14.8 \pm$ & $9.80 \pm$ & $-99.5 \pm$ & 533 & 556.17 & 1.04 \\
& & 0.10 & 0.006 & 11.5 & 14.7 & 74.830 & & & \\
\hline 3.5 & 1.1 & $3.80 \pm$ & $0.10 \pm$ & $32.5 \pm$ & $-25.2 \pm$ & $1868 \pm$ & 533 & 539.2 & 1.01 \\
& & 0.22 & 0.01 & 1.6 & 3.49 & 252 & & & \\
\hline
\end{tabular}

Table 5. BGK fit with valence quarks for $\sigma_{r}$ for H1ZEUS-NC data in the range $Q^{2} \geq 3.5 \mathrm{GeV}^{2}$ and $x \leq 0.01$. NLO fit. Soft + hard gluon. $m_{u d s}=0.14, m_{c}=1.3 \mathrm{GeV}$, non-saturation ansatz. $Q_{0}^{2}=1.9$ or $1.1 \mathrm{GeV}^{2}$.

\begin{tabular}{|c|c|c|c|c|c|c|c|c|c|c|}
\hline$Q_{\min }^{2}\left[\mathrm{GeV}^{2}\right]$ & $Q_{0}^{2}\left[\mathrm{GeV}^{2}\right]$ & $\sigma_{0}[\mathrm{mb}]$ & $A_{g}$ & $\lambda_{g}$ & $C_{g}$ & $D_{g}$ & $E_{g}$ & $N_{d f}$ & $\chi^{2}$ & $\chi^{2} / N_{d f}$ \\
\hline 3.5 & 1.1 & $220 \pm$ & $3.57 \pm$ & $0.082 \pm$ & $31.3 \pm$ & $11.0 \pm$ & $1360 \pm$ & 532 & 532 & 1.00 \\
& & 122 & 0.38 & 0.017 & 5.4 & 9.8 & 690 & & & \\
\hline
\end{tabular}

Table 6. BGK fit with valence quarks for $\sigma_{r}$ for H1ZEUS-NC data in the range $Q^{2} \geq 3.5 \mathrm{GeV}^{2}$ and $x \leq 0.01$.

NLO fit. Soft + hard gluon. $m_{u d s}=0.14, m_{c}=1.3 \mathrm{GeV} . Q_{0}^{2}=1.1 \mathrm{GeV}^{2}$.

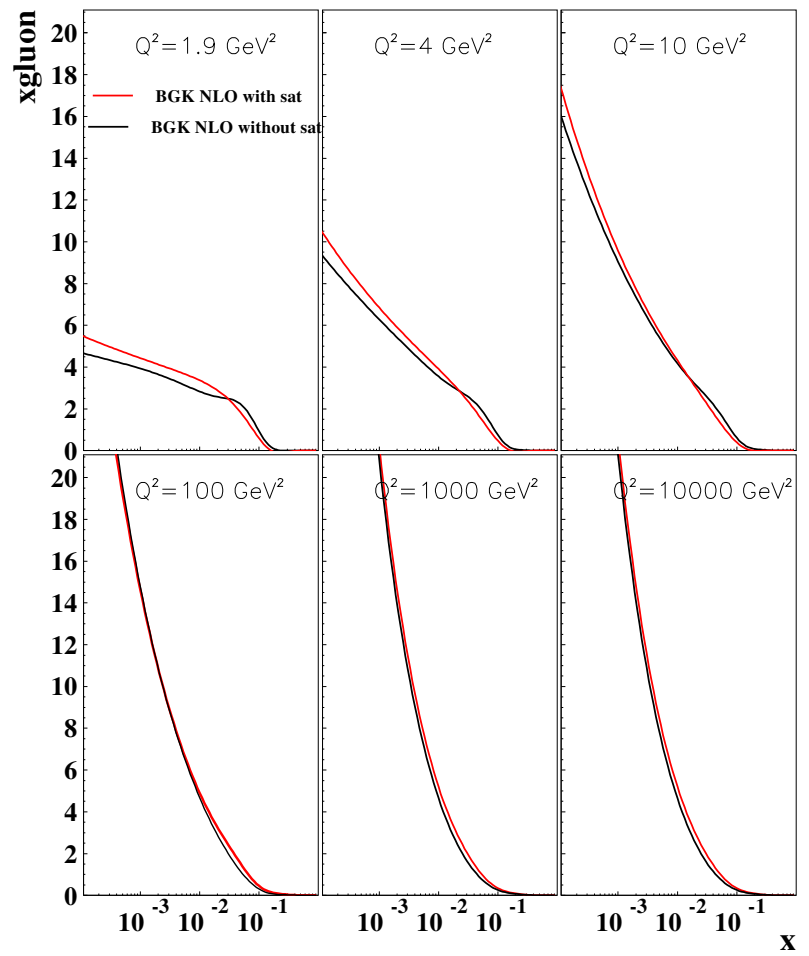

Figure 1. Comparison between the gluon densities obtained from fits in the $Q^{2}>3.5 \mathrm{GeV}^{2}$ region, with and without the saturation ansatz, Table 4 and 5 . 


\section{Predictions with gluon from BGK dipole fit for the LHC}

In Figure 2 we show predictions for the LHC using the gluon density obtained from fits with saturation ansatz of Tables 4 . We see that there is a large discrepancy between ATLAS data for jets and predictions from BGK dipole model.

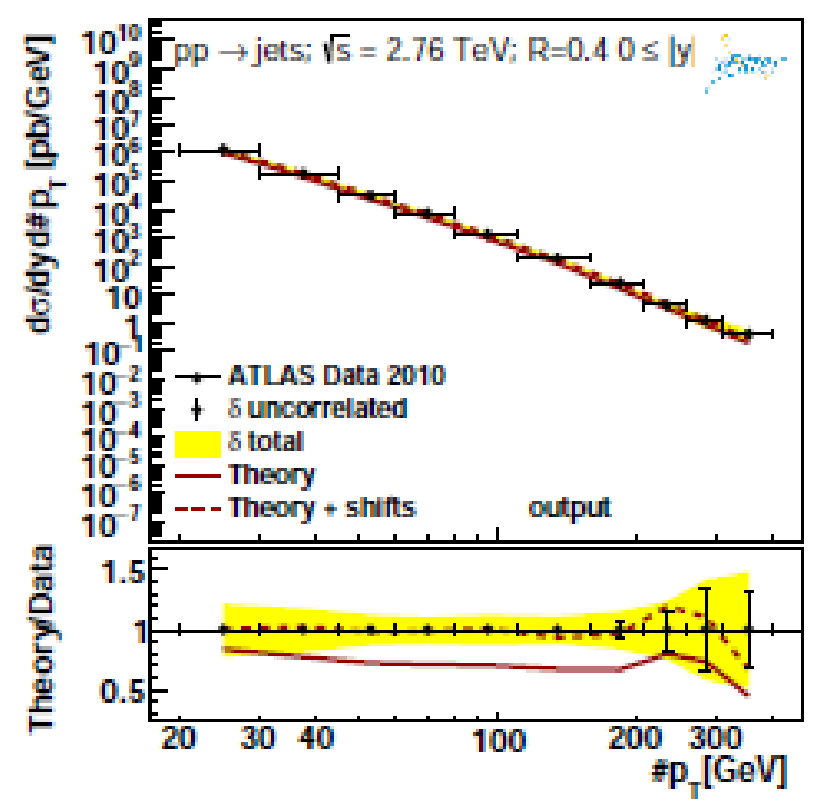

Figure 2. Predictions for jets in ATLAS using gluon density from BGK dipole model

\section{Acknowledgement}

I would like to thank Henri Kowalski (DESY) for various help and careful reading of the manuscript. This work is supported by the Polish Ministry under program Mobility Plus, no. 1320/MOB/IV/2015/0.

\section{References}

[1] K. Golec-Biernat, M. Wuesthoff, Phys. Rev. D59, 014017 (1999); Phys. Rev. D60, 114023 (1999).

[2] J. Bartels, K.Golec-Biernat and H. Kowalski, Phys.Rev. D66, (2002) 19

[3] E. Iancu, K. Itakura, and S. Munier. Phys. Lett., B590:199-208, 2004.

[4] S. Munier, A. M. Staśto and A. H. Mueller, Nucl. Phys. B 603 (2001) 427.

[5] H. Kowalski and D. Teaney, Phys. Rev. D68 (2003) 114005. 
[6] H. Kowalski, L. Motyka and G. Watt, Phys. Rev. D74 (2006) 074016.

[7] F. D. Aaron et al. [H1 and ZEUS Collaborations], JHEP 1001 (2010) 109.

[8] H. Abramowicz et al. [H1 and ZEUS Collaborations], Eur. Phys. J. C75 (2015) nb.12.

[9] "HERAFitter, Open Source QCD Fit Project" By S. Alekhin at al., DESY Report 14-188, 7 Nov 2014, Published in EPJC (2015), 75: 304, e-Print: arXiv:1410.4412 [hep-ex]

[10] N. N. Nikolaev and B. G. Zakharov, Z. Phys. C49 (1991) 607; Z. Phys. C53 (1992) 331.

[11] J. Nemchik, N. N. Nikolaev, E. Predazzi and B. G. Zakharov, Z. Phys. C 75 (1997) 71.

[12] E. Gotsman, E. Levin and U. Maor, Nucl. Phys. B 464 (1996) 251.

[13] H. G. Dosch, T. Gousset, G. Kulzinger and H. J. Pirner, Phys. Rev. D 55 (1997) 2602.

[14] A. C. Caldwell and M. S. Soares, Nucl. Phys. A 696, 125 (2001).

[15] J. R. Forshaw, R. Sandapen and G. Shaw, Phys. Rev. D 69 (2004) 094013.

[16] L. Frankfurt, M. Strikman and C. Weiss, Ann. Rev. Nucl. Part. Sci. 55 (2005) 403.

[17] H. Kowalski, T. Lappi, C. Marquet and R. Venugopalan, Phys. Rev. C78 (2008) 045201. 